\title{
Эффект увеличения фотопроводимости в гетероструктуре II типа $n$-GaSb/InAs/p-GaSb с одиночной квантовой ямой
}

\author{
() М.П. Михайлова, И.А. Андреев, Г.Г. Коновалов, Л.В. Данилов, Э.В. Иванов, Е.В. Куницына, \\ Н.Д. Ильинская, Р.В. Левин, Б.В. Пушный, Ю.П. Яковлев
}

Физико-технический институт им. А.Ф. Иофрфе Российской академии наук, 194021 Санкт-Петербург, Россия

E-mail: Mikh@iropt1.ioffe.ru; Igor@iropt9.ioffe.ru

(Получена 8 февраля 2018 г. Принята к печати 15 февраля 2018 г.)

Сообщается об обнаружении значительного усиления фототока/фотопроводимости при малых обратных смещениях в гетероструктуре II типа $n$-GaSb/InAs/ $p$-GaSb с одиночной квантовой ямой InAs, выращенной методом газофазной эпитаксии из металлоорганических соединений. При воздействии на гетероструктуру монохроматическим излучением с длиной волны $1.2-1.6$ мкм (при $77 \mathrm{~K}$ ) и приложении обратного смещения в диапазоне 5-200 мВ наблюдалось резкое возрастание фототока на 2 порядка. Оптическое усиление зависело от приложенного напряжения и возрастало до значения $2.5 \cdot 10^{2}$ при обратном смещении $800 \mathrm{MB}$. Теоретически установлено, что основную роль в наблюдаемом явлении играет экранирование внешнего электрического поля электронами, локализованными в глубокой квантовой яме, а также туннельный механизм переноса носителей заряда с малой эффективной массой. Показано, что исследуемый эффект усиления фототока является общим для изотипных и анизотипных гетеропереходов II типа, в том числе для структур с квантовыми ямами и сверхрешетками.

DOI: $10.21883 /$ FTP.2018.08.46217.8842

\section{1. Введение}

В последнее десятилетие повышенное внимание исследователей привлекают новые типы фотодетекторов для ближней и средней инфракрасной (ИК) области спектра, в том числе на основе наногетероструктур с глубокими квантовыми ямами (КЯ) и сверхрешетками [1-6]. Такие приборы находят широкое применение в спектроскопии, волоконно-оптических линиях связи, системах экологического мониторинга, газового анализа и медицинской диагностики [2]. При этом актуальной остается задача повышения фоточувствительности и квантовой эффективности фотодиодов на основе узкозонных полупроводников, работающих в ближнем и среднем ИК диапазоне. Поэтому важное значение имеет поиск и исследование новых механизмов усиления фототока в таких детекторах.

Усиление фотосигналов с помощью лавинных фотодиодов требует приложения сильных электрических полей (выше $10^{5} \mathrm{~B} / \mathrm{cm}$ ) для достижения порога ударной ионизации [7]. Применение фототранзисторов требует их сопряжения с малошумящими усилителями [8]. Для создания фоточувствительных приборов с низким энергопотреблением и значительным усилением перспективным представляется использование гетеропереходов II типа в системе GaInAsSb-GaSb, в том числе с КЯ [9].

В гетеропереходах II типа электроны и дырки локализованы по разные стороны гетерограницы. Это обусловливает существование целого ряда уникальных особенностей таких гетеропереходов, проявляющихся в оптических, электролюминесцентных и фотоэлектрических свойствах структур на их основе $[9,10]$. Вследствие малой эффективной массы электронов в такой структуре на границе раздела с широкозонным полупроводником образуется достаточно глубокая КЯ для электронов и формируется туннельно-прозрачный барьер для электронов в зоне проводимости. При освещении созданные излучением неравновесные дырки захватываются в потенциальную ловушку, созданную скачком потенциала в валентной зоне на гетерогранице широкозонного и узкозонного полупроводников. Как было показано в [11-13], в изотипных гетеропереходах аналогичный эффект будет иметь место также в случае захвата дырок на поверхностные состояния, лежащие на гетерогранице.

Ранее нами впервые наблюдался эффект возрастания фототока на 2-3 порядка в объемных изотипных гетеропереходах II типа $n^{+}-\mathrm{GaSb} / n-\mathrm{GaInAsSb} / n^{+}-\mathrm{GaInAsSb}$ с самосогласованными КЯ при малом прямом и обратном смещении в зависимости от интенсивности внешней засветки [14]. Было обнаружено, что небольшое изменение концентрации неравновесных дырок при малых смещениях приводит к резкому изменению туннельной прозрачности квантово-размерного барьера и ток электронов экспоненциально возрастает. Этот эффект был объяснен модуляцией туннельно-прозрачного барьера в зоне проводимости $\mathrm{GaSb}$ за счет локализации неравновесных дырок на гетерогранице и туннелированием электронов через барьер.

В данной работе сообщается об экспериментальном и теоретическом исследовании эффекта значительного фотоэлектрического усиления на примере гетероструктуры $n$-GaSb/InAs/ $p$-GaSb с одиночной квантовой ямой InAs.

\section{2. Технология изготовления структур и методика измерений}

Гетероструктуры $n$-GaSb/InAs/p-GaSb были выращены методом газофазной эпитаксии из металло- 
органических соединений (МОГФЭ) на установке AIXTRON-200 с реактором горизонтального типа на подложках $n-\mathrm{GaSb}: \mathrm{Te}(001)$ с концентрацией электронов $n=(5-7) \cdot 10^{17} \mathrm{~cm}^{-3}$. На подложке последовательно выращивались буферный слой $n-\mathrm{GaSb}: \mathrm{Te}$ (толщина $d=0.8-0.9$ мкм, $\left.n=5 \cdot 10^{17} \mathrm{~cm}^{-3}\right)$, нелегированная одиночная квантовая яма InAs (толщина слоя $d=5$ нм) и накрывающий слой $p-\mathrm{GaSb}: \mathrm{Si}(d=1$ мкм, концентрация дырок $\left.p=(3-4) \cdot 10^{17} \mathrm{~cm}^{-3}\right)[15]$.

Образцы для исследований изготавливались методом стандартной фотолитографии и химического травления в виде диодов с круглой мезой диаметром $\sim 300$ мкм. Омические контакты к подложке $n$-GaSb и накрывающему слою $p$-GaSb были сформированы путем последовательного напыления $\mathrm{Cr} / \mathrm{Au}+\mathrm{Te} / \mathrm{Au}$ или $\mathrm{Cr} / \mathrm{Au}+\mathrm{Ge} / \mathrm{Au}$ соответственно. Образцы размером $500 \times 500$ мкм монтировались на держателях ТО-18 подложкой к корпусу.

Для структур на основе гетеропереходов II типа с одиночной квантовой ямой $n-\mathrm{GaSb} / \mathrm{InAs} / p-\mathrm{GaSb}$ были исследованы вольт-амперные характеристики (BAX), спектральное распределение фоточувствительности, а также зависимость фототока от приложенного напряжения для случев прямого и обратного смещения как при комнатной температуре, так и при температуре жидкого азота. Спектры фоточувствительности исследовались с помощью установки на основе призменного монохроматора SPM-2 с глобаром в качестве источника излучения. Гетероструктуры освещались монохроматическим излучением с длинами волн из области собственного поглощения антимонида галлия со стороны накрывающего слоя $p$-GaSb. Фотосигнал регистрировался методом синхронного детектирования на частоте $\sim 500$ Гц с помощью селективного усилителя SR-830.

\section{3. Результаты эксперимента и обсуждение}

Зонная диаграмма исследуемой гетероструктуры $n$-GaSb/InAs/ $p$-GaSb представляла собой два разьединенных гетероперехода II типа $n$-GaSb/n-InAs и $n$-InAs/ $p$-GaSb, включенных навстречу друг другу (рис. 1). Ранее нами были исследованы спектры электролюминесценции для данной структуры при $T=77 \mathrm{~K}$ [16]. В диапазоне токов накачки от 25 до 150 мА наблюдался возрастающий с током узкий пик с полушириной $\Delta h v=15-20$ мэВ и максимумом в окрестности длины волны $\lambda=3.1$ мкм (энергия фотона $h v=0.41$ эВ), соответствующий излучению из КЯ InAs.

Исследование вольт-амперных характеристик для гетероструктуры $n$-GaSb/InAs/ $p$-GaSb в диапазоне температур $T=77-300 \mathrm{~K}$ показало, что прямая ветвь $\mathrm{BAX}$ $I(U)$ (см. рис. 2) имеет туннельный характер и состоит из трех участков, каждый из которых может быть аппроксимирован экспоненциальной зависимостью $I=I_{0} \exp (e U / \beta k T)$. Коэффициент неидеальности равен $\beta=3-7$ при $T=300 \mathrm{~K}$ и $14-42$ при $77 \mathrm{~K}$. Контактная разность потенциалов, определенная из анализа прямой

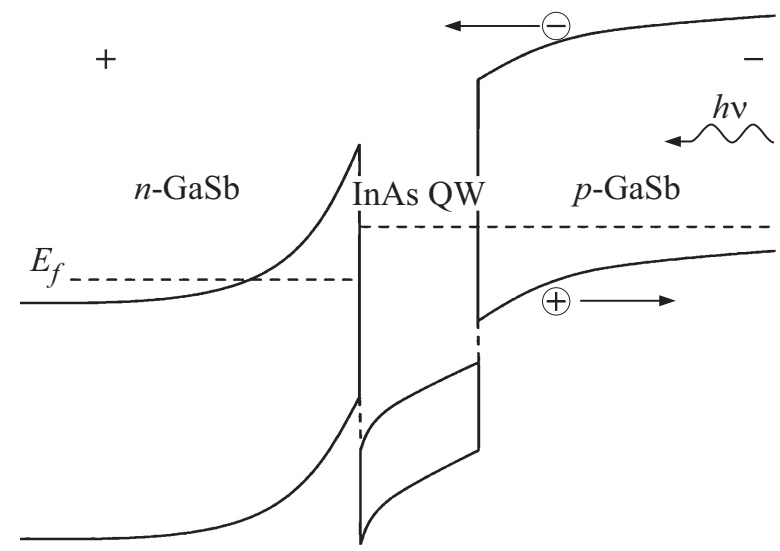

Рис. 1. Зонная диаграмма гетероструктуры $n$-GaSb/InAs/ $p$-GaSb с одиночной квантовой ямой $(\mathrm{QW})$ при обратном смещении. $E_{f}-$ уровень Ферми.

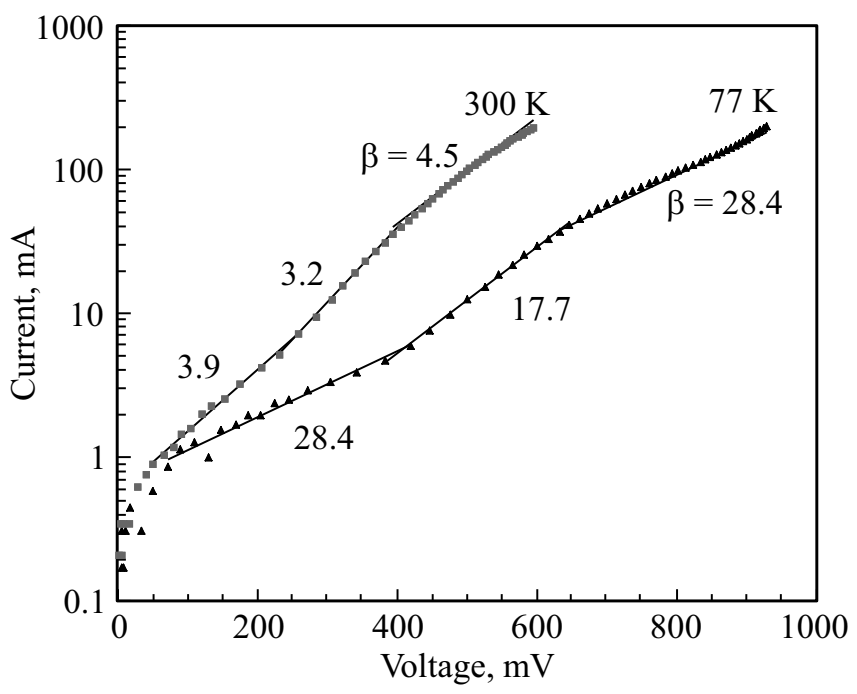

Рис. 2. Вольт-амперные характеристики гетероструктуры $n$-GaSb/InAs/ $p$-GaSb при температурах $T=300$ и $77 \mathrm{~K}$. Точки - эксперимент, отрезки прямых - аппроксимация с помощью формулы $I=I_{0} \exp (e U / \beta k T)$, значения параметра неидеальности $\beta$ указаны.

ветви $\mathrm{BAX}$, составляет $U_{B} \approx 0.4 \mathrm{~B}$ при $300 \mathrm{~K}$ и $0.5 \mathrm{~B}$ при $77 \mathrm{~K}$.

На рис. 3 представлены спектры фотоответа для исследуемой гетероструктуры, измеренные при температуре $77 \mathrm{~K}$ в фотовольтаическом режиме, а также для случая обратного смещения (когда положительный потенциал подан на подложку $n-\mathrm{GaSb}$, отрицательный - на верхний слой $p$-GaSb). Отметим, что при комнатной температуре спектры лежали в диапазоне длин волн 1.0-2.5 мкм с главным максимумом на длине волны $\lambda=1.62$ мкм $(h v=0.77 \ni \mathrm{B})$. Второй, более слабый максимум наблюдался при $\lambda=1.22$ мкм $(h v=1.02$ эВ). По мере уменьшения температуры спектры фотоответа смещались в коротковолновую область, так что при $T=77 \mathrm{~K}$ первый максимум располагался 


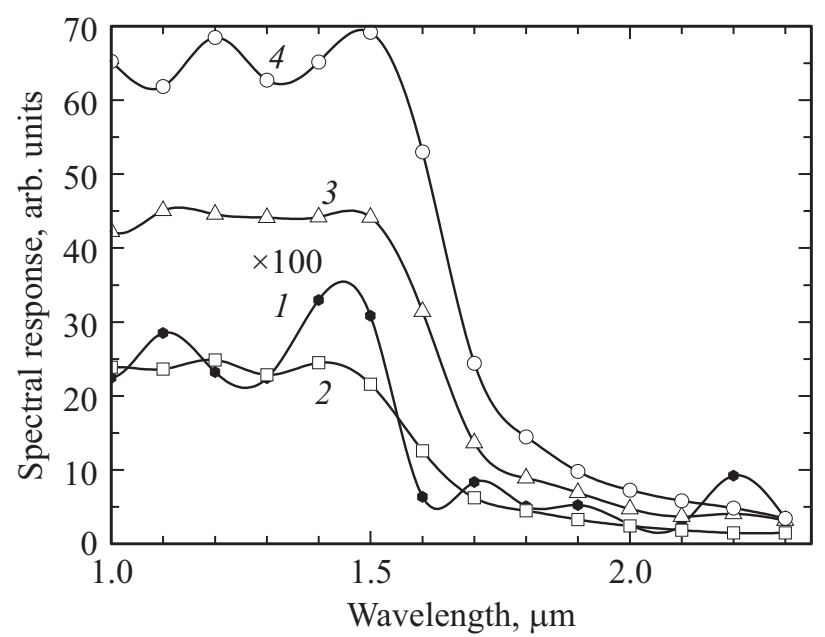

Рис. 3. Спектры фотоответа $(T=77 \mathrm{~K})$ для структуры $n$-GaSb/InAs/ $p$-GaSb в зависимости от величины обратного смещения, B: $1-0,2-0.2,3-0.5,4-0.8$.

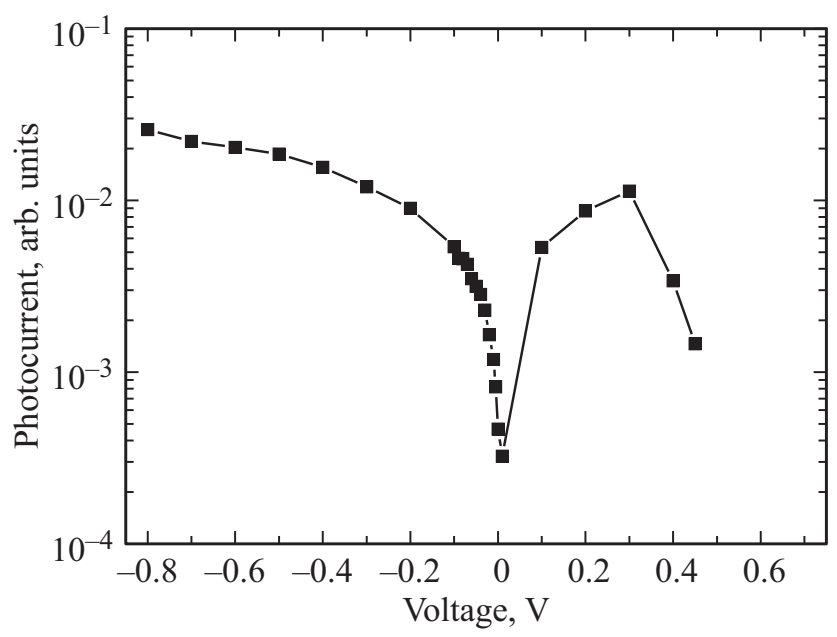

Pис. 4. Зависимость фототока от приложенного напряжения при воздействии на гетероструктуру $n$ - GaSb/InAs/ $p$-GaSb монохроматическим излучением с длиной волны $\lambda=1.5$ мкм при температуре $T=77 \mathrm{~K}$.

в районе $\lambda=1.5$ мкм $(h v=0.83$ эВ), а положение второго максимума определялось длиной волны 1.15 мкм (энергия фотона 1.08 эВ). Температурный коэффициент изменения ширины запрещенной зоны был равен $\Delta E_{g} / \Delta T=-2.7 \cdot 10^{-4}$ эВ $/ \mathrm{K}$, что близко к значению данного параметра для арсенида индия [17].

Область объемного заряда в гетероструктуре $n-\mathrm{GaSb} /$ $\mathrm{InAs} / p$-GaSb расположена главным образом в слое n-GaSb. При температуре жидкого азота энергетическое положение второго максимума в спектре фотоответа $(\lambda=1.15$ мкм $)$ соответствует сумме значений ширины запрещенной зоны для $n$-GaSb и $n$-InAs в структуре $n$-GaSb/InAs/ $p$-GaSb в состоянии термодинамического равновесия, как было показано в работе [9]. Положение главного максимума в спектре фотоответа $(\lambda=1.5$ мкм $)$ определяется возбуждением электронно-дырочных пар в слое $p$-GaSb (см. рис. 1).

На рис. 4 представлена зависимость фототока от приложенного напряжения при монохроматическом освещении исследуемой гетероструктуры с длиной волны $\lambda=1.5$ мкм из области собственного поглощения $\mathrm{GaSb}$ при температуре $77 \mathrm{~K}$. При обратном смещении начиная с малых значений напряжения $(U \approx 5 \mathrm{MB})$ наблюдалось резкое возрастание фототока $I_{\mathrm{ph}}$. При этом в интервале смещений 5-200 мВ коэффициент усиления фототока $G=I_{\mathrm{ph}} / I_{\mathrm{ph} 0}$ достигал значений $G \geq 10^{2}$ (где $I_{\mathrm{ph} 0}-$ величина фототока при обратном смещении $U=5 \mathrm{MB}$ ). По мере увеличения обратного смещения от $200 \mathrm{MB}$ до $0.8 \mathrm{~B}$, наблюдался дальнейший более плавный рост фототока по линейному закону еще на порядок. Этот участок зависимости $I_{\mathrm{ph}}(U)$ соответствует возрастанию фотопроводимости, когда величина коэффициента усиления $G$ определяется соотношением времени жизни и времени пролета для фотоэлектронов. Схожий механизм усиления фототока наблюдался нами ранее в изотипной гетероструктуре $n^{+}$-GaSb/ $n^{0}$-GaInAsSb/ $n^{+}$-GaInAsSb [14]. Таким образом, общее усиление фототока в исследуемом нами гетеропереходе II типа с одиночной квантовой ямой $n-\mathrm{GaSb} / \mathrm{InAs} / p$-GaSb достигало $G \geq 2.5 \cdot 10^{2}$.

Заметное усиление (до $G=40$ ) наблюдалось также при прямом смещении, когда положительный потенциал подан на $p$-GaSb, а отрицательный - на подложку $n$-GaSb, в интервале смещений 5-200 мВ (см. рис. 4). Начиная с напряжения $U \approx 0.3 \mathrm{~B}$, соответствующего значению контактной разности потенциалов $U_{B}$, величина фототока резко уменьшалась.

Обсудим полученные результаты, используя представленную на рис. 1 зонную диаграмму исследуемой структуры $n$-GaSb/InAs/p-GaSb. Вначале рассмотрим случай обратного смещения (рис. $4, U<0$ ). При воздействии на структуру излучением с длиной волны из области собственного поглощения со стороны $p$-GaSb созданные светом неравновесные дырки дрейфуют по направлению к контакту с отрицательной полярностью и захватываются в потенциальную яму в валентной зоне, образованную скачком потенциала $\Delta E_{V}=0.05$ эВ на разъединенной гетерогранице $p$-GaSb/InAs. Фотоэлектроны в свою очередь перемещаются в противоположную сторону, частично захватываются в КЯ на уровень электронов $E_{1}$ и затем резонансно туннелируют через скачок потенциала, образованный изгибом зон на гетерогранице $\mathrm{InAs} / n-\mathrm{GaSb}$. В результате при приложении небольшого обратного смещения фототок резко возрастает.

Ранее значительное усиление фототока было обнаружено в $p-i-n$-гетероструктуре, выращенной на подложке $n^{+}$-InP, со сверхрешеткой $\mathrm{Al}_{0.48} \mathrm{In}_{0.52} \mathrm{As} / \mathrm{Ga}_{0.47} \mathrm{In}_{0.53} \mathrm{As}$ в активной области [18]. При воздействии на данную структуру излучением с энергией фотона больше контактной разности потенциалов фотоответ возрастал на 3-4 порядка. Это явление было объяснено „фильтрацией эффективной массы“ при туннелировании носителей через сверхрешетку за счет большой разницы в эффективных массах электронов и тяжелых дырок. 
Анализируя зависимость $I_{\mathrm{ph}}(U)$ при прямом смещении (рис. 4, $U>0$ ), можно предположить, что в этом случае в процессе туннелирования участвуют легкие дырки. Спад зависимости фототока от прямого смещения при напряжении, большем $U_{B}$, можно объяснить изменением направления фототока по сравнению с темновым током в той точке, где величина положительного смещения сравнивается с контактной разностью потенциалов $\left(U=U_{B}\right)$. Данный эффект наблюдался ранее в работах $[18,19]$, в которых был исследован новый тип фотопроводимости в асимметричной квантовой структуре при резонансном туннелировании через барьер.

\section{4. Теоретические результаты}

Нами был проведен анализ эффекта усиления фотопроводимости в гетероструктуре с одиночной квантовой ямой [20]. Показано, что электростатическое экранирование внешнего электрического поля носителями заряда, локализованными в глубокой КЯ, обусловливает рост дифференциальной фотопроводимости по сравнению с наблюдаемой в объемной $p-i-n$-структур без промежуточного двумерного слоя. С одной стороны, при увеличении обратного напряжения время пролета слоя InAs электронами, генерируемыми при засветке в слое $p$-GaSb, уменьшается, что в свою очередь приводит к уменьшению доли захваченных в квантовую яму электронов. В то же время общий ток электронов через двумерный слой увеличивается, что, напротив, способствует возрастанию концентрации электронов в квантовой яме, а следовательно, способствует увеличению экранирующего электростатического потенциала слоя InAs. Для того чтобы оценить вклад каждого из этих факторов, мы рассмотрели простую модель $p-i-n$-структуры на основе $\mathrm{GaSb}$ с одиночной квантовой ямой InAs в зоне собственной проводимости. В случае, если ширина области собственной проводимости значительно превышает ширину КЯ, напряженность электрического поля в области КЯ можно считать не зависящей от координаты и записать в виде суммы $E=E_{\text {ext }}+E_{\text {int }}+E_{s}$, где первое слагаемое соответствует внешнему электрическому полю, приложенному к гетероструктуре, второе слагаемое - это напряженность внутреннего электрического поля, созданного областями объемного заряда в состоянии термодинамического равновесия, а третье слагаемое учитывает экранирующее поле, создаваемое захваченными в КЯ электронами. Выражение для $E_{s}$ может быть получено из теоремы Гаусса-Остроградского, если квантовую яму представить как бесконечную равномерно заряженную плоскость с плотностью поверхностного заряда $e n^{2 \mathrm{D}}$. В результате имеем

$$
\left|E_{s}\right|=\frac{2 \pi e n^{2 \mathrm{D}}}{\varepsilon} .
$$

Зависимость полного фототока в структуре от напряженности электрического поля выражается в виде

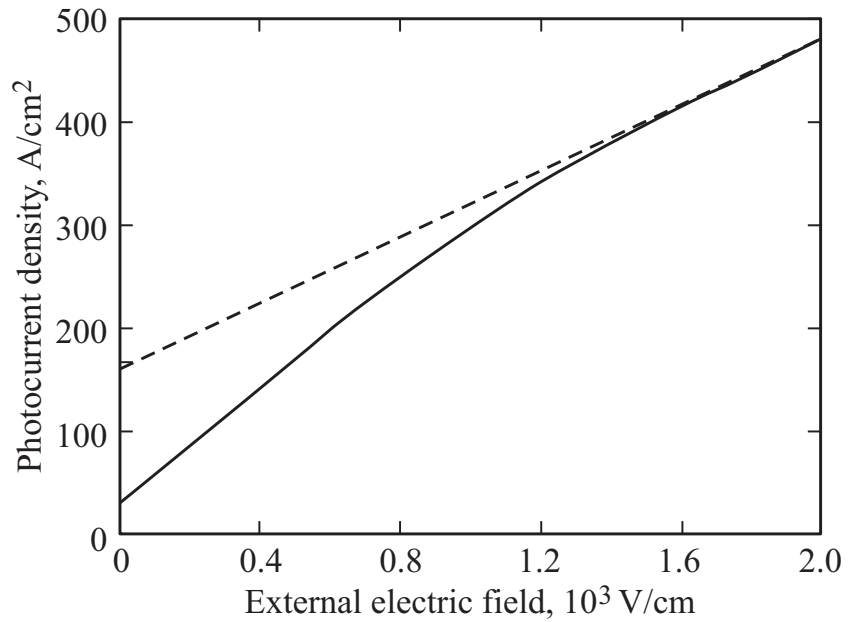

Рис. 5. Теоретическая зависимость плотности фототока от напряженности внешнего электрического поля, приложенного к $p-i-n$-структуре на основе GaSb с одиночной квантовой ямой InAs. Штриховая линия - зависимость фототока от напряженности электрического поля в $p-i-n$-структуре без двумерного слоя InAs.

$j_{0}\left(E_{\mathrm{ext}}\right)=e n_{0} \mu E\left(E_{\mathrm{ext}}\right)$, где $\mu-$ подвижность электронов в $\mathrm{GaSb}, n_{0}=P \tau_{r}$ - концентрация неравновесных электронов на гетрогранице InAs/p-GaSb, определяемая интенсивностью засветки $P$ и временем жизни носителей заряда $\tau_{r}$ в слое $p$-GaSb. В свою очередь напряженность электрического поля $E$ будет зависеть от концентрации электронов, локализованных в КЯ, которая входит в слагаемое $E_{s}$. Таким образом, мы имеем самосогласованную задачу, где $n^{2 \mathrm{D}}$ неявным образом зависит от $E_{\text {ext }}$.

Для того чтобы найти зависимость концентрации электронов в КЯ $n^{2 \mathrm{D}}$ от напряженности внешнего электрического поля необходимо сопоставить выражения для тока электронов, захватываемых в КЯ, и туннельного тока электронов из КЯ через диффузионный барьер, образованный областью объемного заряда в $n-\mathrm{GaSb}$,

$$
j_{0}\left\{1-\exp \left[-\frac{d}{\mu \tau E\left(E_{\text {ext }}\right)}\right]\right\}=e \tilde{T}\left(k_{x}\right) v_{x} \frac{n^{2 \mathrm{D}}}{d},
$$

где $\tilde{T}\left(k_{x}\right)$ - коэффициент туннельной прозрачности, зависящий от квазиимпульса электронов в направлении $x$, перпендикулярном плоскости гетерограницы, величина $v_{x}$ соответствует $x$-компоненте скорости электронов, локализованных в квантовой яме вблизи гетерограницы $n$-GaSb/InAs, $m_{c}$ - эффективная масса электрона в InAs.

Получив зависимость $n^{2 \mathrm{D}}\left(E_{\text {ext }}\right)$, нетрудно построить зависимость плотности полного фототока в структуре от напряженности внешнего электрического поля, $j_{0}\left(E_{\mathrm{ext}}\right)$, которая представлена на рис. 5. Как видно из данной зависимости, поведение плотности фототока аналогично тому, что наблюдалось в эксперименте (см. рис. 4). Построенную теоретическую зависимость можно приближенно аппроксимировать двумя линейными участками с различными углами наклона. Первый, более резкий, 


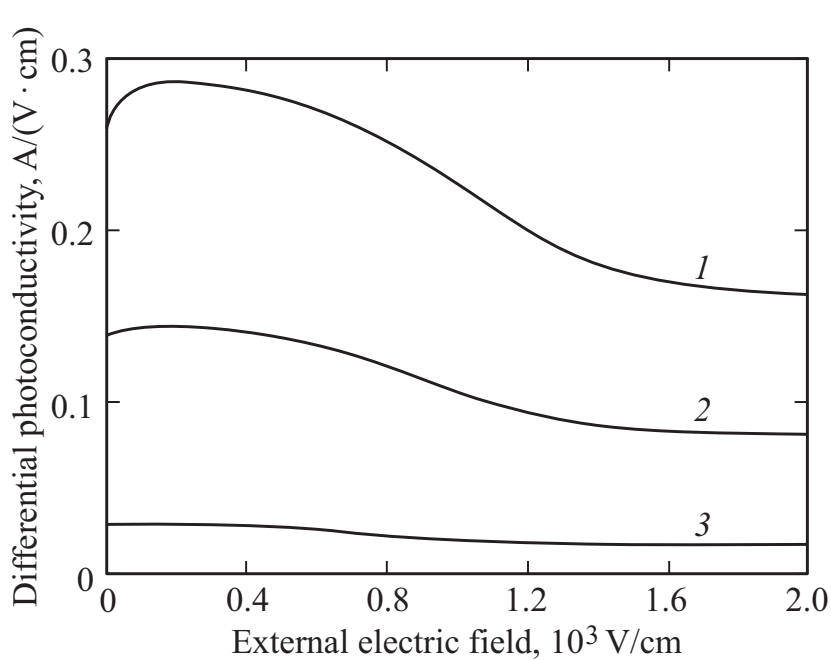

Рис. 6. Теоретическая зависимость дифференциальной фотопроводимости от внешнего электрического поля в $p-i-n$ структуре на основе $\mathrm{GaSb}$ с одиночной квантовой ямой InAs для концентраций неравновесных носителей заряда $n_{0}=10^{14}(1), 5 \cdot 10^{14}(2), 10^{15} \mathrm{~cm}^{-3}(3)$.

участок от нуля до $1000 \mathrm{~B} / \mathrm{cm}$ соответствует фототоку в условиях электростатического экранирования электронами, локализованными в КЯ, и определяется в основном туннельным током через диффузионный барьер на гетерогранице $n$-GaSb/InAs. Второй участок с напряженностью приложенного поля > $1000 \mathrm{~B} /$ см соответствует случаю, когда фототок полностью определяется дрейфовым током электронов через двумерный слой без захвата в КЯ. При этом мы имеем обычную резистивную зависимость фототока от напряженности внешнего электрического поля, характерную для объемных $p-i-n$-структур (на рис. 5 она показана штриховой линией).

Для того чтобы оценить фоточувствительность структуры с промежуточным двумерным слоем по сравнению с обычной объемной $p-i-n$-структурой, необходимо построить зависимость дифференциальной фотопроводимости, определяемой выражением $\gamma\left(E_{\text {ext }}\right)=d j_{0} / d E_{\text {ext }}$, от напряженности внешнего электрического поля.

На рис. 6 представлены кривые дифференциальной фотопроводимости для различных концентраций неравновесных носителей заряда, возбужденных в слое $p$-GaSb благодаря внешней засветке. Видно, что рассматриваемая фотодиодная структура, работающая при малых внешних смещениях, когда значительная доля фотоэлектронов захватывается в КЯ, имеет в $1.5-2$ раза более высокую дифференциальную фотопроводимость по сравнению со структурой, работающей в резистивном режиме при больших внешних смещениях. Таким образом, можно заключить, что фотодиодные гетероструктуры с промежуточным двумерным слоем имеют существенно более высокую фоточувствительность по сравнению с объемными $p-i-n$-структурами, которая к тому же достигается при достаточно малых внешних смещениях.
Оценка ампер-ваттной чувствительности для исследованной гетероструктуры $n$-GaSb/InAs/p-GaSb на длине волны $\lambda=1.5$ мкм при температуре $77 \mathrm{~K}$, сделанная методом сравнения с чувствительностью стандартного фотодиода на основе $p-n$-перехода $\mathrm{GaSb}$ без КЯ, работающего в фотовольтаическом режиме с эффективностью $0.6 \mathrm{~A} / \mathrm{B}$, дает значение $4.2 \cdot 10^{2} \mathrm{~A} / \mathrm{B}$.

В заключение отметим, что эффекты усиления фототока можно наблюдать в гетероструктурах II типа с глубокими КЯ и высокими потенциальными барьерами на основе таких соединений, как $\mathrm{GaSb} / \mathrm{InAs}(\mathrm{Sb}), \mathrm{GaSb} / \mathrm{AlSb}$, $\mathrm{InAs}(\mathrm{Sb}) / \mathrm{Al}(\mathrm{As}) \mathrm{Sb}$. Основной причиной возрастания фотопроводимости в указанных гетероструктурах является туннельный механизм переноса носителей заряда, что приводит к „фильтрации эффективной массы“ [18], т.е. перенос заряда осуществляется преимущественно носителями с малой эффективной массой (электронами или легкими дырками), а также „залипание“ дырок на скачке потенциала в валентной зоне широкозонного полупроводника $p$-GaSb, что приводит к модуляции туннельнопрозрачного барьера на гетерогранице широкозонного и узкозонного соединений.

\section{5. Заключение}

Впервые наблюдался эффект увеличения фотопроводимости при малом обратном смещении в гетероструктуре II типа $n-\mathrm{GaSb} / \mathrm{InAs} / p$-GaSb с одиночной квантовой ямой и двумя разъединенными гетеропереходами. Для объяснения данного явления был проведен теоретический анализ процессов переноса и захвата носителей заряда в исследуемой гетероструктуре, который показывает необходимость учета эффекта электростатического экранирования внешнего электрического поля электронами, локализованными в квантовой яме InAs.

Наблюдаемый механизм усиления фототока в наноструктурах $n$-GaSb/InAs/ $p$-GaSb с разъединенными гетеропереходами II типа на интерфейсах является беспороговым, в отличие от процесса ударной ионизации, идущего с высоким порогом, $\sim(3 / 2) E_{g}$, поскольку значительное усиление фототока достигается при малом обратном смещении.

Эффект увеличения фотопроводимости в полупроводниковых структурах с гетерограницами II типа, по нашему мнению, является общим для изотипных и анизотипных гетеропереходов (как ступенчатых, так и разъединенных) и может наблюдаться также и на других материалах $\mathrm{A}^{\mathrm{III}} \mathrm{B}^{\mathrm{V}}, \mathrm{A}^{\mathrm{II}} \mathrm{B}^{\mathrm{VI}}, \mathrm{A}^{\mathrm{IV}} \mathrm{B}^{\mathrm{IV}}$, квантовых ямах и сверхрешетках.

Данное явление может быть использовано для улучшения параметров фотодетекторов ближнего и среднего ИК диапазона на основе сверхрешеток и гетероструктур II типа. Поскольку высокий уровень фоточувствительности достигается при малых внешних смещениях, обнаруженный эффект также представляет интерес с точки 
зрения разработки высокоскоростных коммуникационных систем с низким энергопотреблением.

\section{Список литературы}

[1] Y. Wei, A. Hood, H.Yau, A. Gin, M. Razeghi, M.Z. Tidrow, V. Nathan. Appl. Phys. Lett., 86 (23), 233106 (2005).

[2] M. Mikhailova, N. Stoyanov, I. Andreev, B. Zhurtanov, S. Kizhaev, E. Kunitsyna, K. Salikhov, Yu. Yakovlev. Proc. SPIE, 6585 (Optical Sensing Technology and Applications), 658526 (2007).

[3] M. Razeghi, D. Hoffman, B.-M. Nguyen, P.-Y. Delaunay, E.K. Huang, M.Z. Tidrow. Proc. SPIE, 6940 (Infrared Technology and Applications XXXIV), 694009 (2008).

[4] P.K.D.D.P. Pitigala, Y.F. Lao, A.G.U. Perera, L.H. Li, E.H. Linfield, H.C. Liu. J. Appl. Phys., 115 (6), 063105 (2014).

[5] M. Ahmetoglu, B. Kucur, I.A. Andreev, E.V. Kunitsyna, M.P. Mikhailova, Yu.P. Yakovlev. Acta Phys. Polon. A, 127 (4), 1007 (2015).

[6] Е.В. Куницына, Е.А. Гребенщикова, Г.Г. Коновалов, И.А. Андреев, Ю.П. Яковлев. ФТП, 50 (10), 1420 (2016).

[7] Ф. Капассо. В сб.: Техника оптической связи: Фотоприемники, под ред. У. Тсанга (М., Мир, 1988) гл. 1.

[8] Дж. Кэмпбелл. В сб.: Техника оптической связи: Фотоприемники, под ред. У. Тсанга (М., Мир, 1988) гл. 5.

[9] M.P. Mikhailova, A.N. Titkov. Semicond. Sci. Technol., 9 (7), 1279 (1994).

[10] В.A. Wilson. IEEE J. Quant. Electron., 24 (8), 1763 (1988).

[11] А.Д. Милнс, Д.Л. Фойхт. Гетеропереходы и переходы металл-полупроводник (М., Мир, 1975) гл. 4.

[12] C. van Opdorp, J. Vrakking. Solid-State Electron., 10, 995 (1967).

[13] S. Yawata, R.L. Anderson. Phys. Status Solidi, 12, 297 (1965).

[14] И.А. Андреев, А.Н. Баранов, М.А. Мирсагатов, М.П. Михайлова, А.А. Рогачев, Г.М. Филаретова, Ю.П. Яковлев. Письма ЖТФ, 14 (5), 389 (1988).

[15] Р.В. Левин, В.Н. Неведомский, Б.В. Пушный, Н.А. Берт, М.Н. Мизеров. Письма ЖТФ, 42 (2), 79 (2016).

[16] L.V. Danilov, M.P. Mikhailova, E.V. Ivanov, G.G. Konovalov, E.A. Grebenschikova, R.V. Levin, B.V. Pushnyi, G.G. Zegrya, Yu.P. Yakovlev. Proc. 24th Int. Symp. Nanostructures: Physics and Technology (St. Petersburg, Russia, 2016) p. 219.

[17] Handbook Series on Semiconductor Parameters, ed. by M. Levinstein, S. Rumyantsev, M. Shur (Singapore-N. Y.London-Hong Kong, World Scientific Publishing, 1996) v. 1, ch. 6.

[18] F. Capasso, K. Mohammed, A.Y. Cho, R. Hull, A.L. Hutchinson. Phys. Rev. Lett., 55 (10), 1152 (1985).

[19] C. Sirtori, J. Faist, F. Capasso, D.L. Sivco, A.Y. Cho. Appl. Phys. Lett., 63 (19), 2670 (1993).

[20] Л.В. Данилов, М.П. Михайлова, И.А. Андреев, Г.Г. Зегря. ФТП, 51 (9), 1196 (2017).

Редактор Л.В. Шаронова

\section{The photoconductivity amplification phenomenon in the type II $n$-GaSb/InAs/ $p$-GaSb heterostructure with a single quantum well}

M.P. Mikhailova, I.A. Andreev, G.G. Konovalov,

L.V. Danilov, E.V. Ivanov, E.V. Kunitsyna, N.D. Ilinskaya, R.V. Levin, B.V. Pushnyi, Yu.P. Yakovlev

loffe Institute, 194021 St. Petersburg, Russia

Abstract We report the observation of a significant amplification phenomenon of the photocurrent/photoconductivity at low reverse bias in the type II $n$-GaSb/InAs/ $p$-GaSb heterostructure with a single quantum well grown by metallorganic vapor phase epitaxy. Large increase of the photocurrent reached the value more than two orders at $77 \mathrm{~K}$ under illumination of the samples by monochromatic radiation with the wavelength of $1.2-1.6 \mu \mathrm{m}$ at the reverse bias of 5-200 $\mathrm{mV}$. The optical gain was sensitive to the applied voltage and increased up to $2.5 \cdot 10^{2}$ at the reverse bias of $800 \mathrm{mV}$. This phenomenon was theoretically explained by the screening of external electric field by electrons accumulated in the InAs quantum well, as well as tunneling of the charge carriers with a small effective mass. It is shown that the effect under study is inherent in both isotype and anisotype type II heterojunctions, including the structures with quantum wells and superlattices. 